\title{
Esophagogastroduodenoscopy Patient
}

National Cancer Institute

\section{Source}

National Cancer Institute. Esophagogastroduodenoscopy Patient. NCI Thesaurus. Code C159857.

A patient who is undergoing an esophagogastroduodenoscopy. 